\title{
Triboelectric Nanogenerator as a Self-powered Communication Unit for Processing and Transmitting Information
}

\author{
Aifang Yu, ${ }^{\dagger, \perp}$ Xiangyu Chen,,${ }^{\dagger, \perp}$ Rui Wang,${ }^{\dagger, \perp}$ Jingyu Liu, ${ }^{\dagger}$ Jianjun Luo, Libo \\ Chen, ${ }^{\dagger}$ Yang Zhang, ${ }^{\dagger}$ Wei Wu,${ }^{\dagger}$ Caihong Liu, ${ }^{\dagger}$ Hongtao Yuan, ${ }^{\dagger}$ Mingzeng Peng, ${ }^{\dagger}$ \\ Weiguo Hu ${ }^{\dagger, *}$, Junyi Zhai ${ }^{\dagger, *}$ and Zhong Lin Wang ${ }^{\dagger, \S, *}$
}

$\dagger$ Beijing Institute of Nanoenergy and Nanosystems, Chinese Academy of Sciences, China

$\S$ School of Materials Science and Engineering, Georgia Institute of Technology, Atlanta, Georgia 30332-0245,United States.

$\perp$ A.-F. Yu, X.-Y. Chen, and R. Wang contributed equally to this work.

Address correspondence to huweiguo@binn.cas.cn(WGH), jyzhai@binn.cas.cn (JYZ) and zlwang@gatech.edu (ZLW) 

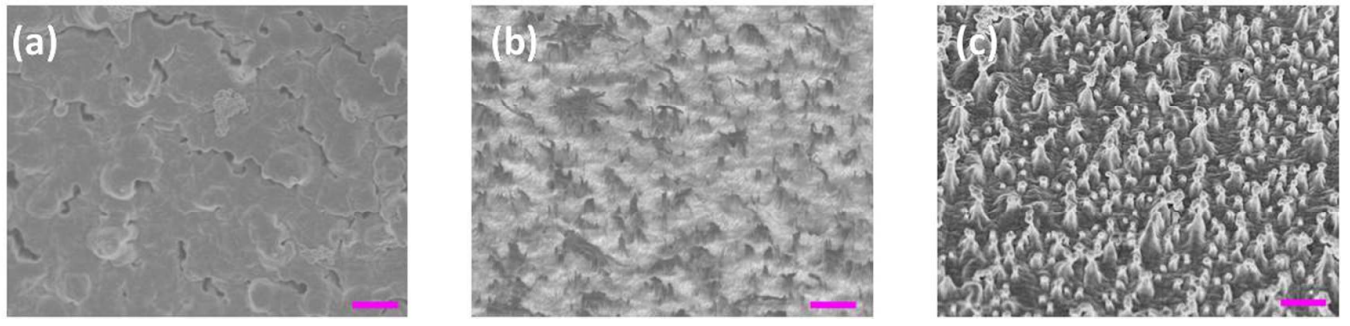

Figure S1. (a) Thick, short, and rare microwires. (b) Rare clusters of nanowires. (c) Denseand uniform nanowires. The bar is $1 \mu \mathrm{m}$.

Figure S1 gives the SEM morphology of the PTFE contact surface. The microstructures on the PTFE surface were fabricated using inductively coupled plasma (ICP) reactive ion etching. In the optimized etching process, a $\mathrm{Cu}$ film with a thickness of approximately $40 \mathrm{~nm}$ was deposited on the PTFE surface as the mask. A mixed gas including $\mathrm{Ar}, \mathrm{O}_{2}$, and $\mathrm{CF}_{4}$ was introduced in the ICP chamber, where the corresponding flow rates were 15.0, 10.0, and $30 \mathrm{sccm}$, respectively. The nanostructure was etched under a power source of $200 \mathrm{~W}$ to generate a large density of plasma, and the time was $30 \mathrm{~min}$. Therefore, the nanowires in Figure S1c were compact and uniform, which was totally different from that in Figure S1a and Figure S1b. In Figure S1a, an etching process without the mask was used, and the etching time was $20 \mathrm{~min}$. The thickness of the mask was $20 \mathrm{~nm}$, and the etching time was 30 min in Figure S1b. 


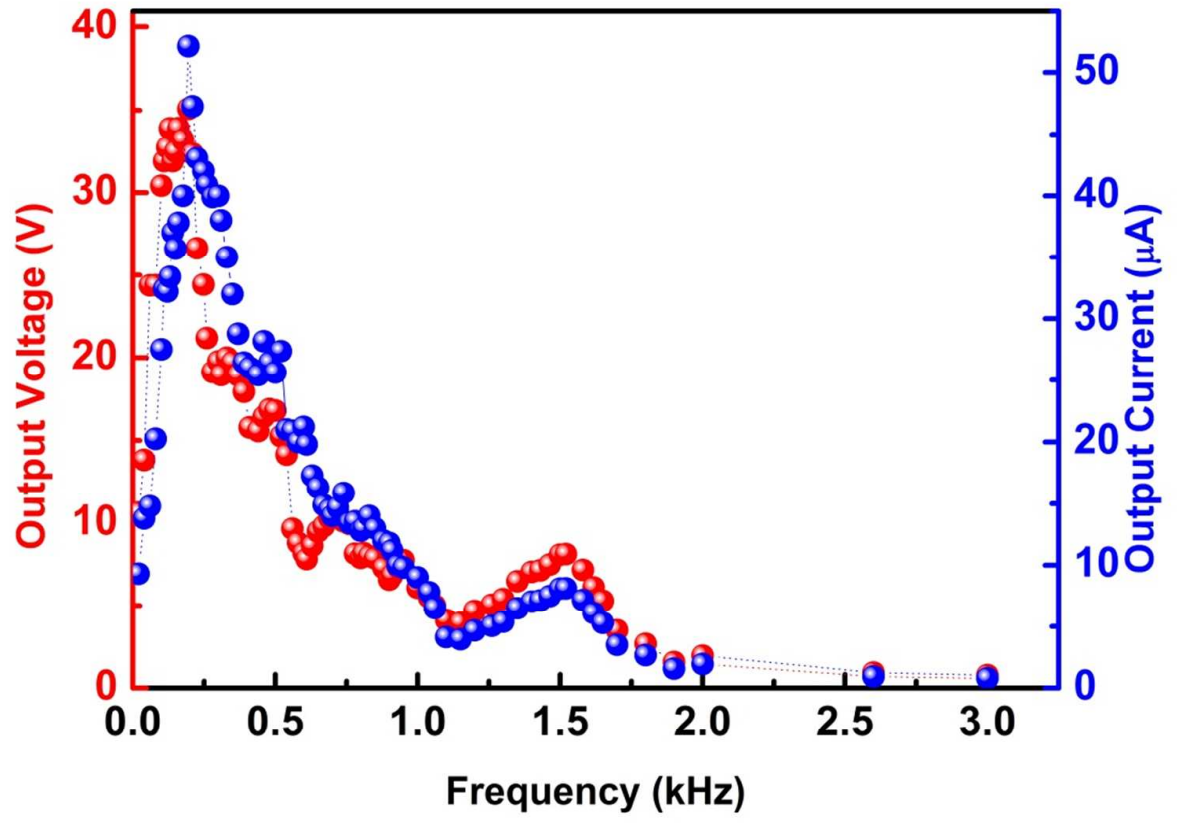

Figure S2. Relationship between frequency and electrical output of as-fabricated TENG at bands from $0.02 \mathrm{kHz}$ to $3.0 \mathrm{kHz}$. 
(a)
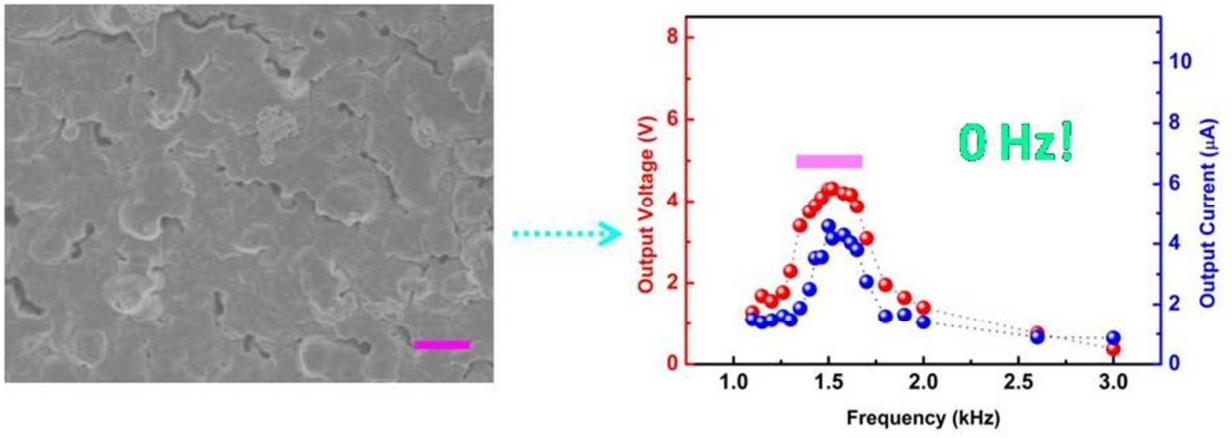

(b)
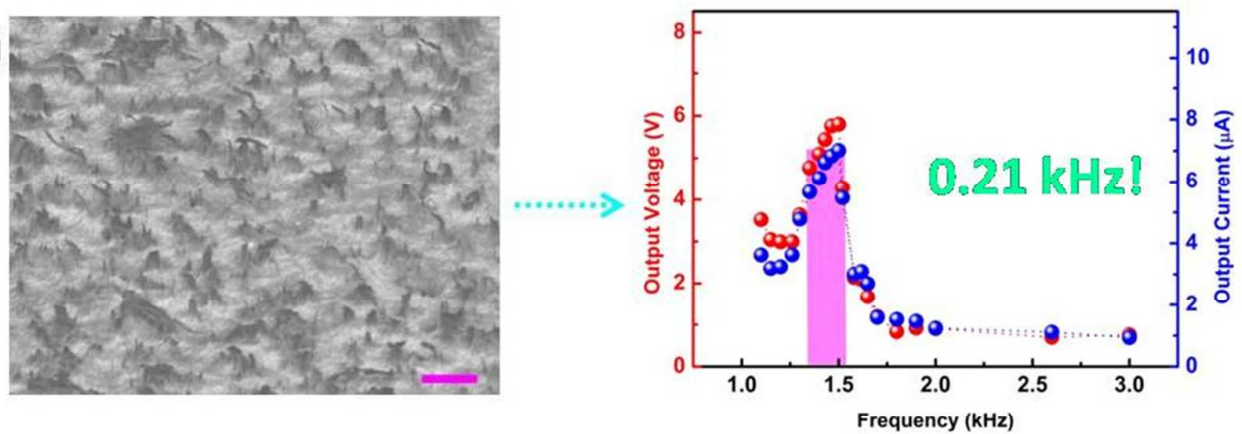

(c)

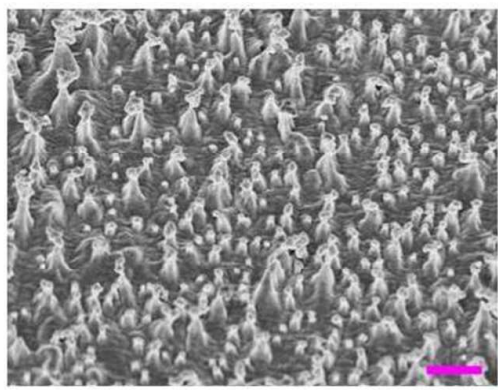

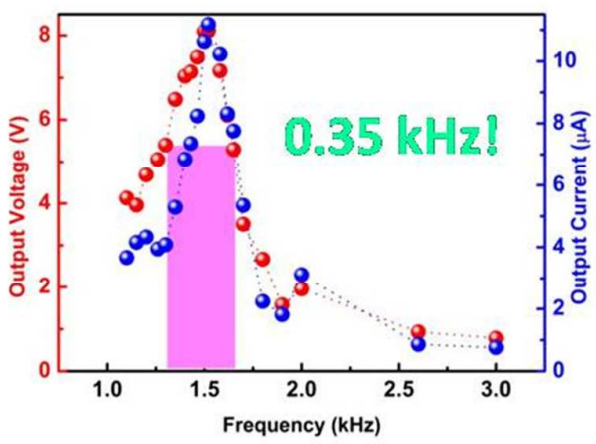

Figure S3. The frequency responses of TENG with three different microstructures. 

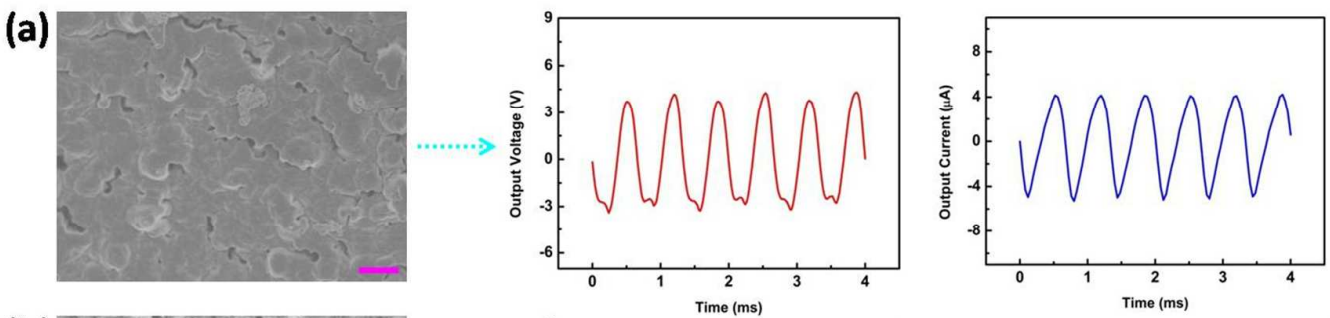

(b)
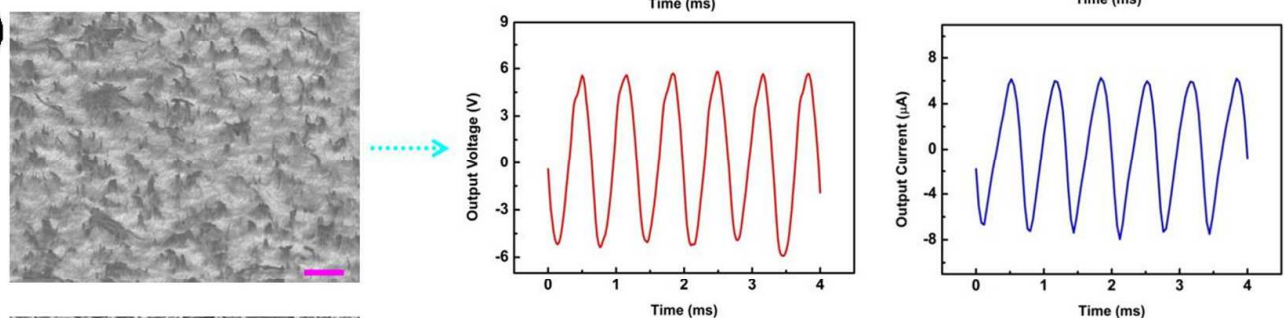

(c)
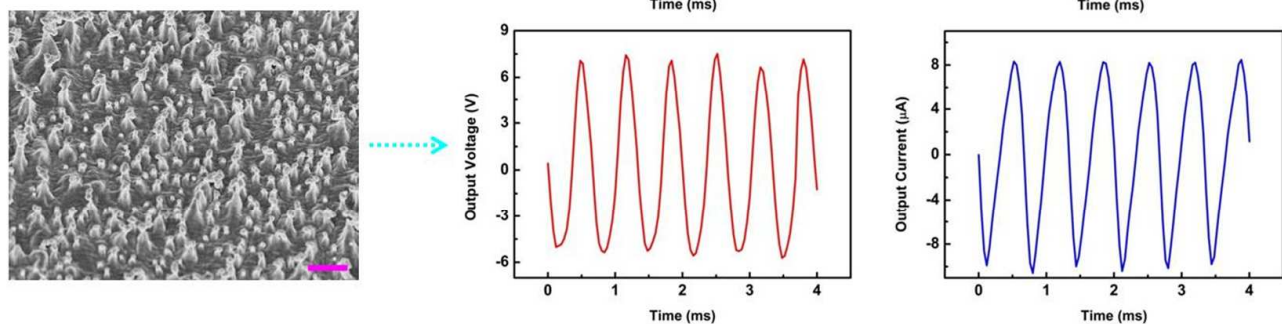

Figure S4. The output voltage and output current of TENG with three different microstructures.

The output performance of TENG based on these nanostructures has exhibited significant differences, as shown in Figure S4. The intensity of the input sound was approximately $95 \mathrm{~dB}$ in all measurements in this study. As discussed in Refs.1-5, the highest output in Figure S4c can be attributed to the dense and uniform nanowires, which significantly increased the surface roughness and the effective surface area of the TENG for effective tribo-electrification. In this self-powered communication system, the output generated from the TENG should be enough to drive the LED and to excite the photoresistor. In order to overcome all the energy consumptions of the system, the minimum output voltage and current of the system must be larger than $5 \mathrm{~V}$ and $4.2 \mu \mathrm{A}$, respectively. These values were also the threshold values for the TENG 
devices. Therefore, only the outputs in Figure S4b and Figure S4c can successfully transmit information. The communication system driven by TENG with an output shown in Figure S4a does not normally work, which proved the necessity of the surface modification method. Of course, the high output can provide more accurate information transmission. On the other hand, the microstructure also influenced the bandwidth of communication, as shown in Figure S3. For communication, the wider the bandwidth is, the faster the communication velocity is. In conclusion, interfacial nanostructures on the contact surface were vital for the performance of TENG, and nanofabrication represented the core technology of TENG. Thus, optimized nanostructures were chosen to construct the TENG in the text. 


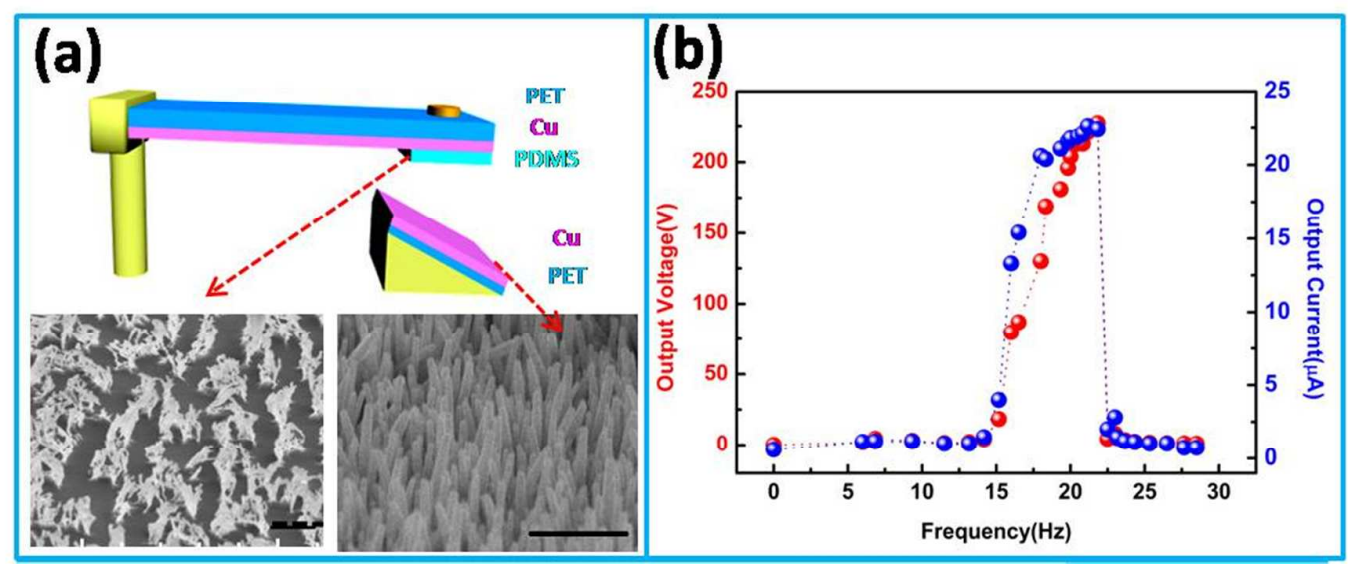

Figure S5. (a) Structure and (b) relationship between frequency and electrical output of as-fabricated TENG with a cantilever structure. The bar is $1 \mu \mathrm{m}$.

\section{Reference}

1. Fan, F.R.; Lin, L.; Zhu, G.; Wu, W.; Zhang, R.; Wang, Z. L. Transparent Triboelectric Nanogenerators and Self-Powered Pressure Sensors Based on Micropatterned Plastic Films. Nano Lett. 2012, 12, 3109-3114.

2. Zhu, G.; Lin, Z.-H.; Jing, Q.; Bai, P.; Pan, C.; Yang, Y.; Zhou, Y.; Wang, Z. L. Toward Large-Scale Energy Harvesting by a Nanoparticle-Enhanced Triboelectric Nanogenerator. Nano Lett. 2013, 13, 847-853.

3. Jeong, C. K.; Baek, K. M.; Niu, S.; Nam, T. W.; Hur, Y. H.; Park, D. Y.; Hwang, G.-T.; Byun, M.; Wang, Z. L.; Jung, Y. S. Topographically-Designed Triboelectric Nanogenerator via Block Copolymer Self-Assembly. Nano Lett. 2014, 14, 7031-7038.

4. Feng, Y.; Zheng, Y.; Ma, S.; Wang, D.; Zhou, F.; Liu, W. High Output Polypropylene Nanowire Array Triboelectric Nanogenerator Through Surface Structural Control and Chemical Modification. Nano Energy 2016, 19, 48-57.

5. Li, H. Y.; Su, L.; Kuang, S. Y.; Pan, C. F.; Zhu, G.; Wang, Z. L. Significant Enhancement of Triboelectric Charge Density by Fluorinated Surface Modification in Nanoscale for Converting Mechanical Energy. Adv. Funct. Mater. 2015, 25, 5691-5697.

6. Seung, W.; Gupta, M. K.; Lee, K. Y.; Shin, K.-S.; Lee, J.-H.; Kim, T. Y.; Kim, S.; Lin, J.; Kim, J. H.; Kim, S.-W. Nanopatterned Textile-Based Wearable Triboelectric Nanogenerator. ACS Nano 2015, 9, 3501-3509. 
7. Yun, B. K.; Kim, J. W.; Kim, H. S.; Jung, K. W.; Yi, Y.; Jeong, M.-S.; Ko, J.-H.; Jung, J. H. Base-Treated Polydimethylsiloxane Surfaces as Enhanced Triboelectric Nanogenerators. Nano Energy 2015, 15, 523-529.

8. Ha, M.; Park, J.; Lee, Y.; Ko, H. Triboelectric Generators and Sensors for Self-Powered Wearable Electronics. ACS Nano 2015, 9, 3421-3427.

9. Chen, J.; Zhu, G.; Yang, J.; Jing, Q.; Bai, P.; Yang, W.; Qi, X.; Su, Y.; Wang, Z. L. Personalized Keystroke Dynamics for Self-Powered Human-Machine Interfacing. ACS Nano 2015, 9, 105-116.

10. Yeh, M.-H.; Lin, L.; Yang, P.-K.; Wang, Z. L. Motion-Driven Electrochromic Reactions for Self-Powered Smart Window System. ACS Nano 2015, 9, 4757-4765.

11. Quan, T.; Wang, X.; Wang, Z. L.; Yang, Y. Hybridized Electromagnetic-Triboelectric Nanogenerator for a Self-Powered Electronic Watch. ACS Nano 2015, 9, 12301-12310.

12.Hu, Y. F.; Yang, J.; Jing, Q. S.; Niu, S. M.; Wu, W. Z.; Wang, Z. L. Triboelectric Nanogenerator Built on Suspended 3D Spiral Structure as Vibration and Positioning Sensor and Wave Energy Harvester. ACS Nano 2013, 7, 10424-10432.

13. Tang, W.; Tian, J.; Zheng, Q.; Yan, L.; Wang, J.; Li, Z.; Wang, Z. L. Implantable Self-Powered Low-Level Laser Cure System for Mouse Embryonic Osteoblasts' Proliferation and Differentiation. ACS Nano 2015, 9, 7867-7873.

14. Zhang, C.; Tang, W.; Zhang, L.; Han, C.; Wang, Z. L. Contact Electrification Field-Effect Transistor. ACS Nano 2014, 8, 8702-8709.

15. Chun, J.; Kim, J. W.; Jung, W.-s.; Kang, C.-Y.; Kim, S.-W.; Wang, Z. L.; Baik, J. M. Mesoporous Pores Impregnated with $\mathrm{Au}$ Nanoparticles as Effective Dielectrics for Enhancing Triboelectric Nanogenerator Performance in Harsh Environments. Energ. Environ. Sci. 2015, 8, 3006-3012. 